about prescribing practices, and invited doctors, all above F1 training level, to complete this by email.

We emailed 120 doctors and received 72 completed questionnaires; $52.1 \%$ of the respondents were female, $53.4 \%$ had more than 6 years' experience as a doctor and $66.0 \%$ had prescribed to non-patients. Of that last group, 93.3\% did not inform the person's regular general practitioner, with 95.0\% feeling it was unnecessary to do so. The most commonly prescribed medications were antibiotics $(77.3 \%)$, followed by analgesics (25.0\%) and the oral contraceptive pill (18.2\%). Of note, a number of respondents stated that they had prescribed sleeping pills (16.8\%) and smoking cessation medications (8.5\%).

Most doctors felt it appropriate to prescribe antibiotics, analgesics and inhalers, and some felt it was acceptable to prescribe the oral contraceptive pill and antipsychotic medication, to family and friends; $58.9 \%$ admitted to selfprescribing.

Although the majority of doctors had used private prescriptions, approximately a fifth had used National Health Service prescriptions (21\%). Finally, 55.3\% reported never reading the GMC guidelines on prescribing.

Our results show that a large proportion of doctors are not adhering to GMC guidelines on medication prescribing. In many cases this may be attributable to simply not reading the guidelines. We suggest that the GMC considers publicising its prescribing guidance more widely to ensure good medical practice and to avoid the consequences of escalating poor prescribing habits.

1 Press Association. GMC finds Osborne brother guilty. The Guardian, 22 February 2010 (http://www.guardian.co.uk/uk/feedarticle/8957107).

2 General Medical Council. Good Practice in Prescribing Medicines Guidance for Doctors. GMC, 2008 (http://www.gmc-uk.org/guidance/ ethical_guidance/prescriptions_faqs.asp).

Lubna Karim, Psychiatric Trainee, The Royal Free Hospital, email: lubna.karim@doctors.org.uk, Golnar Aref-Adib, General Practice Vocational Training Scheme, Barnet Hospital, Apu Chakraborty, Consultant Psychiatrist, The Royal Free Hospital, London.

doi: $10.1192 / \mathrm{pb} .34 .8 .356 a$

\section{Three consultants for one patient}

Singhal et al concluded that communication between consultants is vital but is not necessarily the key to success in provision of service for patients. The model in their study quite rightly looked at the role of two key workers (consultants), but did not look at the provision of care for patients in the intervening period between discharge from hospital and follow-up appointments with the community mental health team (CMHT) consultant. The crisis resolution home treatment team (CRHTT) plays a vital role in this intervening period. In an evaluation of our services, we found $44 \%$ of patients are now discharged into the CRHTT. The teams are obliged to care for these patients until their mental state is sufficiently stable for safe and effective transfer to the CMHT, and this period of intervention varies from a few days to several weeks. In effect, with the New Ways of Working, ${ }^{2}$ over a third of patients with an in-patient stay would have received care from three different consultants. While the patient is under the care of the CRHTT there may be changes to the overall care plan including changes to psychotropic medication. For these patients it is then three consultants for one patient and maybe four consultants if they have comorbid drug and alcohol dependence as well. It is therefore not surprising that most patients are not aware of the demarcations between the services. Communication and sharing of information with service users and their carers is as important as it is between two or more consultants and their teams.

Of the 170 mental health professionals who participated in Singhal et al's study, only two were from the liaison service. In our experience of working in a CRHTT, some patients were unaware of the role of the consultant despite being fully informed by the team. It is not unusual for patients to request to remain permanently under the care of the CRHTT. Singhal et al's suggestion that there is a need for a larger nationwide study is necessary and most welcome. Although the jury is still out on the advantages and disadvantages of two consultants for one patient, the current process of service provision for a significant number of patients involves a third consultant in the CRHTT, and we recommend that further studies should seek the views of mental health professionals and service users who received care from a third consultant. Crisis resolution home treatment teams have to a large extent filled the gap created by New Ways of Working with regard to continuity of care and their role in provision of service should not be overlooked.

1 Singhal A, Garg D, Rana AK, Naheed M. Two consultants for one patient service users' and service providers' views on 'New Ways'. Psychiatrist 2010: 34: 181-6.

2 Department of Health. Mental Health: New Ways of Working for Everyone. Department of Health, 2007.

Kishen Neelam, ST6 in General Psychiatry, Greater Manchester West Mental Health NHS Foundation Trust, email: kishen.neelam@yahoo.co.uk, Fola Williams, Trust Consultant Psychiatrist, Crisis Resolution and Home Treatment Service, Salford.

doi: $10.1192 / p b .34 .8 .357$ 